\title{
Phenomenology of visuo-spatial working memory task performance
}

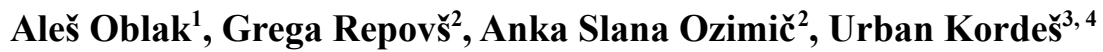 \\ ${ }^{1}$ Laboratory for Cognitive Neuroscience and Psychopathology, University Psychiatric Hospital Ljubljana, \\ Ljubljana, Slovenia \\ ${ }^{2}$ Mind \& Brain Lab, Department of Psychology, Faculty of Arts, University of Ljubljana, Ljubljana, \\ Slovenia \\ ${ }^{3}$ Center for Cognitive Science, Faculty of Education, University of Ljubljana, Ljubljana, Slovenia \\ ${ }^{4}$ Observatory: Laboratory for Empirical Phenomenology, Faculty of Education, University of Ljubljana, \\ Ljubljana, Slovenia
}

\begin{abstract}
In experimental cognitive psychology, objects of inquiry have typically been operationalized with psychological tasks. If we are interested in measuring the target phenomena, we must inquire into the validity of the task; that is, to what extent does the task elicit the phenomenon in question. If we subscribe to the second view, evaluating the validity and the interpretation of the gathered data can be supplemented by understanding the experience of solving psychological tasks. The aim of the present article is to investigate how individuals experience performing a psychological task, specifically, a visuo-spatial working memory task. We present ethnographic descriptions of different ways individuals can experience the same task. We focus on aspects of experience that comprise the overall sense of experience (e.g., bodily feelings, emotional atmosphere, mood). We discuss the methodological implications of our findings and the possibility of conducting a neurophenomenology of visuo-spatial working memory.
\end{abstract}

Keywords: visuo-spatial working memory, empirical phenomenology, neurophenomenology, psychological task, intersubject variability

\section{Introduction}

In experimental cognitive psychology, objects of inquiry have typically been operationalized with psychological tasks ${ }^{1}$. Many readers will likely have been participants in studies using such tasks and have sat in front of a computer screen displaying that typical bright gray background, waiting for stimuli to appear. In well-established domains of research, these tasks are standardized, allowing them to be readily modified to address specific research questions. We can interpret data gathered with psychological tasks in two ways. First, we can choose to limit our interpretation to participants' behavior rather than include in our inquiry what they experienced. In other words, psychological tasks can be thought of as isolating behavioral measures rather than phenomenology. Second, we can posit that the psychological tasks elicit

\footnotetext{
${ }^{1}$ We adopt the term psychological task based on Morrison et al. (2019), where it refers to a task in everyday sense as something that is to be done, but deployed in the context of psychological research to isolate and make measurable some phenomenon.
} 
the desired cognitive phenomenon (cf. Morrison et al., 2019). According to the former view (i.e., measuring behavior alone) we are concerned with the reliability of our instrument when assessing our findings. Conversely, if we are interested in measuring the target phenomena, we must inquire into the validity of the task; that is, to what extent does the task elicit the phenomenon in question. If we subscribe to the second view, evaluating the validity and the interpretation of the gathered data can be supplemented by understanding the experience of solving psychological tasks (cf. Jaspers, 1997). The aim of the present article is therefore to investigate how individuals experience performing a psychological task, specifically, a visuo-spatial working memory task.

Seghier and Price (2018) propose that the same psychological tasks can be solved with several different cognitive strategies. Additionally, psychological tasks are commonly accompanied by boredom (D'Angiulli \& Smith LeBeau, 2002), anxiety (Ikeda et al., 1996), and mind-wandering (Morrison et al., 2019). These factors are associated with intersubjective variability, that is, differences in measurements between participants. Intersubjective variability can be associated with several extraneous variables. One possibility that has remained under-examined is that participants experience the same tasks in different ways. That is, not only is it possible that participants solve the same task using different strategies, but experience the same task differently, even when the strategies they employ remain similar.

Two studies used contemporary approaches for gathering so-called phenomenal data (Varela \& Shear, 1999) to address the questions of intersubjective variability. First, it has been shown that different strategies, as accessible in conscious reflection, are associated with different electrophysiological signatures during simple visual task performance (Lutz et al., 2002). Second, elicited and spontaneously occurring inner speech are related not only to different but opposite patterns of neural activation as recorded by fMRI (Fernyhough et al., 2018; Hurlburt et al., 2016). While working memory has been studied from a phenomenological point of view (Buschsbaum, 2013), to the best of our knowledge, novel approaches in first-person research have not been applied to this phenomenon. Using these methods might help us disentangle intersubjective variability in visuo-spatial working memory research.

This paper is part of a larger project on the phenomenology of visuo-spatial working memory. Different aspects of the experience of engaging with a visuo-spatial working memory task, for example, what strategies participants use to solve such a task and how working memory is experienced in semi-naturalistic settings (Oblak, 2020) are presented elsewhere. This paper is structured as follows: First, we provide a detailed overview of the research design (Section 2). Second, we present ethnographic descriptions of different ways individuals can experience the same task (Subsection 3.1). Third, we provide the results of the qualitative analysis of gathered first-person data, whose aim was to overview the main types of experience associated with visuo-spatial working memory task performance (Subsection 3.2). We focus on an aspect of experience we term background feelings (Section 4). Background feelings refer to aspects of experience that comprise the overall sense of experience (e.g., bodily feelings, emotional atmosphere, mood) (cf. Colombetti, 2011). Finally, we relate our findings to extant phenomenological insights (Section 5.1.), and present some of the methodological lessons of this study on how they could be integrated within a neuroscientific framework (Section 5.2).

\section{Materials and Methods}

A combination of a computerized behavioral task designed to investigate visuo-spatial working memory and an in-depth phenomenological interview was used to gather first-person data. Participants were asked to attend four interview sessions. During each session, participants were asked to solve multiple trials of the visuo-spatial working memory task. At a random moment during task-performance, participants were prompted to report on their experience. A phenomenological interview followed. The interview 
investigated both how participants' experience evolved through time during the trial immediately before the prompt, as well as what they experienced during each moment of the trial. Additionally, behavioral data on their task-performance (i.e., performance accuracy and reaction times) were gathered but are not reported in this paper.

Audio recordings of the interviews were analyzed according to the principles of constructivist grounded theory. Our main analytical instrument was coding (i.e., assigning general descriptive tags to sections of raw data). Three stages of coding took place. First, codes were induced solely from the data. Second, extant categories were compared to as-of-yet uncoded data, while novel categories were induced. Third, all the categories were fitted to the data. In line with principles of qualitative research, data acquisition and analysis were conducted in parallel, one informing the other. Then, relational coding was used to establish logical relationships between individual experiential categories constructed via coding. Finally, a codebook was constructed, outlining both individual experiential categories, and the relationships between them. In a subset of participants, the resulting codebook was validated in additional interviews.

Figure 1 provides a schematic outline of the research design. Each aspect of the research design is presented in detail in subsequent subsections.

\section{A/ Data acquisition}

Sampling sessions $\mathrm{x} 4$

\begin{tabular}{|l|l|l|} 
Visuo-spatial workingmemory task performance & Prompt to report & Phenomenological interview
\end{tabular}

B/ Qualitative analysis

Inductive coding

\begin{tabular}{c}
$\begin{array}{c}\text { Audio recordings of } \\
\text { interviews }\end{array} \longrightarrow \begin{array}{c}\text { Experiential categories induced from } \\
\text { the data }\end{array}$ \\
\hline
\end{tabular}

Inductive-deductive coding

\begin{tabular}{|c|c|c|}
\hline $\begin{array}{c}\text { Fitting extant } \\
\text { categories to the data }\end{array}$ & $\begin{array}{l}\text { Audio recordings of } \\
\text { interviews }\end{array}$ & $\begin{array}{l}\text { Experiential categories inducedfrom } \\
\text { the data }\end{array}$ \\
\hline
\end{tabular}

Deductive coding

Fitting extant
categories to the data $\longrightarrow \begin{gathered}\text { Audio recordings of } \\ \text { interviews }\end{gathered}$

Relational coding

Experiential categories

Constructing a codebook

C/ Validation

Fifth session

\begin{tabular}{|l|l|l|} 
Visuo-spatial workingmemory task performance & Prompt to report & Reportw/ experiential categories
\end{tabular}

Figure 2. Structure of the research design.

\subsection{Participants}


31 participants ( 18 females) aged between 20 and 50 years $(M=27.0, S D=6.21$; years of education: $M=$ $16.52, S D=2.35$ ) signed an informed consent to participate in four 60-minute study sessions in which they performed a visuo-spatial working memory task. All participants had normal or corrected-to-normal eyesight. All but three participants were right-handed. The sample size was determined based on conceptual depth of the data (see Section 2.4., Analysis), and by the expectation that it would match or exceed the sample size in a typical working memory study (usually, between 20 and 30 participants, e.g., Bo \& Seidler, 2009). 16 participants were students of cognitive science at the University of Ljubljana. Nine of them participated in the study as a part of their coursework. Since their participation was therefore not strictly speaking voluntary, they were given the option for their data to not be used in the analysis. None of the participants opted for having their data removed. To avoid recruiting only from the student body, additional 15 participants who had no background in mind sciences were recruited. The nine participants who were involved in the study as a part of the course in first-person research received course credits. For non-student participants no reward was given in exchange.

\subsection{Instruments}

\subsubsection{Visuo-spatial Change Detection Task}

The participants completed multiple trials of the visuo-spatial change detection task, in which they were asked to memorize orientations, colors or positions of the presented stimuli. The timing structure of each trial is presented in Figure 2. Briefly, in the remember color and remember orientation conditions, four target stimuli were presented simultaneously for $2.0 \mathrm{~s}$. In the remember position, the target stimuli were presented sequentially, each for the duration of $0.75 \mathrm{~s}$. Following a maintenance delay of $2.0 \mathrm{~s}$, the probe stimuli were shown, and the participants had to indicate by a button press whether there was a change in the relevant property in any of the stimuli. Both the accuracy and reaction times of the responses were recorded. If the participant did not respond within $2.5 \mathrm{~s}$, a null response was recorded, and the participant proceeded to a next trial. 


\section{A/ Temporal structure}

Color \& orientation condition

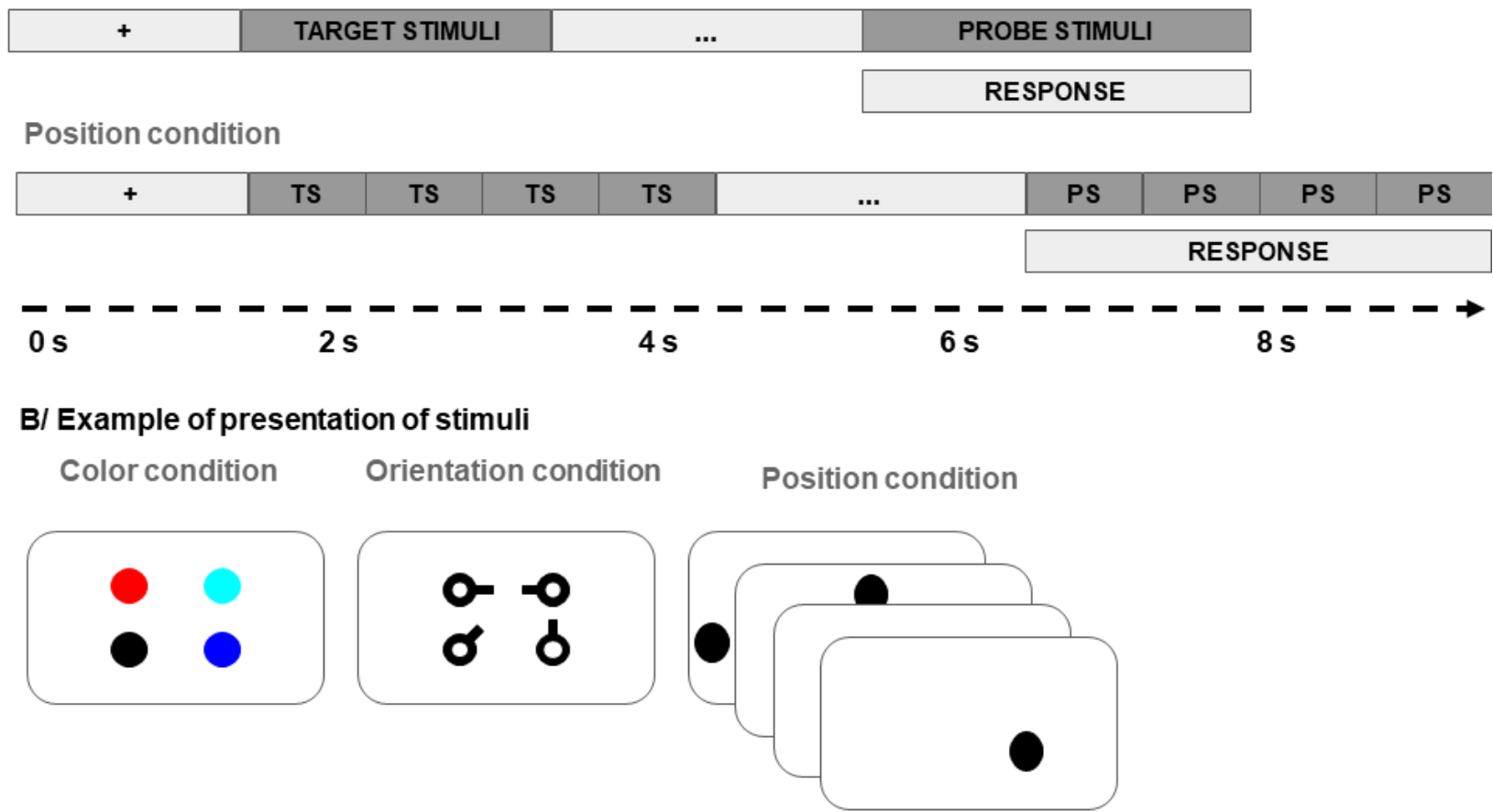

Figure 2: Timing structure and conditions in visuo-spatial change detection task. A: timing structure, B: Example of presentation of target and probe stimuli in color, orientation and position conditions. TS - target stimuli, PS - probe stimuli. Stimuli sizes in the figure are approximations. For precise dimensions, consult the text below.

The visuo-spatial change detection task was presented on an Acer Aspire 3 laptop (Intel Core i5 processor with $2.50 \mathrm{GHz}$ and $3 \mathrm{MB}$ RAM) running Windows 10 Pro operating system. The stimuli were presented on a 15.6-inch full LED screen with $1920 \times 1080$ resolution and $60 \mathrm{~Hz}$ refresh rate. The screen was set to maximum brightness $\left(224 \mathrm{~cd} / \mathrm{m}^{2}\right)$ when the task was being performed. Participants sat approximately 75 $\mathrm{cm}$ away from the center of the screen.

A custom script prepared in PsychoPy (Peirce, 2007) was used to present the stimuli and collect responses. Stimuli in the orientation condition were black keys (see Figure 2), $35 \mathrm{px}\left(0.55^{\circ}\right.$ visual angle) in length, with the main circle $19 \mathrm{px}\left(0.3^{\circ}\right.$ visual angle $)$ in diameter and the line $7 \mathrm{px}\left(0.11^{\circ}\right.$ visual angle $)$ wide. The orientation of each stimulus was randomly selected from eight possible principal directions pointing towards $0^{\circ}, 45^{\circ}, 90^{\circ}, 135^{\circ}, 180^{\circ}, 225^{\circ}, 270^{\circ}$ and $315^{\circ}$. Stimuli in the color condition were colored circles, $35 \mathrm{px}\left(0.55^{\circ}\right.$ visual angle $)$ in diameter. The color of each stimulus was randomly selected from eight easily distinguishable color hues: red, dark blue, light blue, green, yellow, purple, and white. In the position condition, the stimuli were black circles, $20 \mathrm{px}\left(0.32^{\circ}\right.$ visual angle $)$ in diameter. 
Stimuli in all conditions were presented on a grey background within an invisible square bounding box $520 \mathrm{px}\left(8.14^{\circ}\right.$ visual angle $)$ in width positioned in the center of the screen. The position of the orientation and color stimuli was fixed in the vertices of the invisible square. The exact position of the stimuli within the square varied randomly between trials, with the requirement that the minimal distance between the centers of each pair of objects be at least 4 times their bounding radius.

\subsubsection{The Phenomenological Interview}

The automated change-detection task was designed to allow pausing after any trial and allow for experiential sampling. Specifically, between the seventh and the fifteenth trial, the interviewer paused task execution after participants responded to the probe stimuli. The interviewer then asked the participants to stop performing the task and reflect on their experience. The interviewer guided them through the empirical phenomenological interview in which participants' subjective reports were gathered.

The interview was designed to address the research question (i.e., how participants experience solving the visuo-spatial working memory task). It integrated interviewing approaches from qualitative research-specifically, constructivist grounded theory (Charmaz, 2004; Charmaz \& Belgrave, 2007; Mills et al., 2006a; 2006b)-and extant empirical-phenomenological approaches, such as second-person in-depth phenomenological inquiry (Kordeš \& Klauser, 2016), micro-phenomenological interview (Petitmengin, 2006), and descriptive experience sampling (Hurlburt, 2011).

Constructivist grounded theory and second-person in-depth phenomenological inquiry are both approaches that consider the constructive role of both the researcher and the participants in the process of knowledge-creation. The methods require participants to be highly engaged with the study. In turn, the interested position of the participants-unlike some contemporary approaches to first-person research (e.g., Petitmengin, 2006; Hurlburt, 2011)-allow the participants to go beyond an open-ended style of interviewing. Rather, focused questions allowing for further theory-construction may be asked (cf. Charmaz, 2004).

The descriptive experience sampling technique is based on randomly sampling experiential episodes of individuals who are trained to observe and report their experience. We modified the typical descriptive experience sampling approach, which is based on sampling experience in an ecological setting, by inducing the target experience (i.e., solving a visuo-spatial working memory task) in a laboratory setting. 
Initially, the interviewer asked about the overall temporal structure of the participants' experience: what were the salient events that took place in their awareness and what was their temporal succession relative to the behavioral and functional structure of the working memory task (i.e., presentation of the target stimuli/encoding, delay/maintenance, probe stimuli/recall and response). Then, the interviewer guided the participant back to the earliest moment they reported on and gathered its detailed description. The interviewer guided the participants away from general statements, descriptions of their beliefs about their experience, folk-psychological theories, and scientific (psychological and phenomenological) concepts (Petitmengin et al., 2019; Valenzuela-Moguilansky \& Vásquez-Rosati, 2019). When descriptions of all salient experiences were grounded in bodily feelings, mental gestures, specific sensory modalities, and/or attitudes, the interviewer guided the participants to the next event. When the participant reported that there is nothing more to add regarding the trial, they were invited to return to the working memory task.

Audio recordings of the interviews were gathered using an Olympus WS-852 digital voice recorder.

\subsection{Procedure}

Participants were asked to take part in four study sessions, each lasting 60 minutes. The four sessions took place within two weeks. Data were collected in an empty classroom with a table sectioned off for research purposes. During the task performance, the interviewer sat behind the participants.

During the first session, after signing the consent form, the participants were told how to perform the task. This included instructions for the visuo-spatial change detection task, as well as how to observe their experience (i.e., disregarding scientific and folk psychological theories, assumptions, and beliefs). Prior to the beginning of each session, the interviewer provided a verbal description of both the working memory task and the interviewing protocol. It was emphasized that the participants should set aside their assumptions about the nature of their experience and cognition and focus only on the events and processes that occur in their awareness.

During each session, two task conditions were tested. One condition was concluded at the 30-minute mark of the session. To avoid overwhelming participants with too many task parameters, the stimuli with simultaneous presentation (color and orientation) were presented at the first session. Within it, half of the participants started with the color condition and half with the orientation condition. Sequential presentation (position) was introduced in the first part of the second session. In the next session, conditions were randomized. When a new task condition was introduced, the participants were given a sample trial. The procedure was repeated until an hour elapsed. ${ }^{2}$

\footnotetext{
${ }^{2}$ It may be suspected that sampling multiple experiences within a single interview session prompted participants towards employing one single strategy; that is, the refractory period between the samplings when they had to reflect on their experience biased them towards using the same strategy. Due to the nature of our method, we lack a sufficient number of samples to confirm this suspicion. However, qualitative insights present in Subsection 3.2. suggest that a) strategies of task-performance are of secondary importance; and b) that how often participants shifted their strategies largely depends on their overall dispositions towards the task.
} 
A preliminary qualitative analysis of each session took place within 24 hours after the interview. These analyses informed what follow-up questions were asked in subsequent interviews. In other words, data acquisition and initial data analysis were conducted in parallel. The process of parallel analysis allowed us to focus on the salient aspects of experience during the interview process itself and narrow our focus on the data directly addressing our research question (cf. Charmaz, 2004; Flick, 2009; Kordeš \& Klauser, 2016).

\subsection{Data analysis}

A total of 124 sessions were conducted across all the participants. All in all, we gathered 501 samples of experience (on average, 16.2 per participant). On average, participants received 4.0 prompts per session. A handful of participants expressed a higher degree of interest in exploring their experience. Sessions with them consisted of fewer samples that were explored in more detail.

Based on current standards in first-person research (Hurlburt, 2011; Vasquez-Rosatti \& Valenzuela-Moguillansky, 2019), two criteria for determining the validity of individual samples were followed: a) whether the participants focused their description on a single trial; and b) whether they described their lived experience rather than theories, assumptions, or beliefs) (e.g., statements such as "I simply saved it in my working memory." or "I stored it in my brain."). If a given sample did not reach these criteria, we eliminated the sampled experience from the analysis. Commonly, first sessions were eliminated entirely, as were various samples that did not conform to the above-mentioned criteria. 376 samples (133 for the color condition, 134 for the orientation condition, and 109 for the position condition) of the experience of solving the change-detection task were considered valid and were analysed further.

\subsubsection{Analysis of the interviews}

Interview data were analyzed according to the principles of constructivist grounded theory. The main analytical instrument in constructivist grounded theory is coding; that is, the process of assigning more general descriptive tags to sections of raw data (see Subsection 2.4.1.1.). Codes were grouped together based on their descriptive similarities, resulting in many experiential categories (see Subsection 3.2.), The analysis yielded a large taxonomy of experiential categories. Thus, to facilitate the understanding of first-person data, representative ethnographic descriptions were constructed, aiming to provide an intuitive overview of the data (see Subsection 3.1.).

\subsubsection{Coding}

During the analysis, the samples from all participants and across the three tasks were grouped together. Valid samples were coded according to the principles of constructivist grounded theory (Charmaz, 2004). Our goal was to determine a system of classification that would fully describe the key aspects of experience associated with the performance of a change-detection task. Initially, our focus was on the explicit ways of how individuals solve the change detection task - what we can broadly refer to as 
"strategies." However, as the data were coded inductively, other experiential categories that will be in detail presented below emerged through the process of analysis. ${ }^{3}$

Following inductive coding, we employed relational coding, i.e., we constructed meaningful relationships between individual codes (Flick, 2009). Most importantly, we grouped them into higher-order categories based on descriptive similarities. Considering the large amount of phenomenal data acquired, we constructed a broad taxonomy of experiences associated with solving a change detection task (e.g., the most well-developed codes span five levels of abstraction). It is important to note here that the data we initially gathered were both broad and detailed (e.g., we gathered descriptions of how participants experienced their mental space taking shape in their consciousness). In relational coding, we narrowed our focus and constructed experiential categories that are explicitly related to engaging with a psychological task, and specifically, solving a visuo-spatial working memory task. We omitted those aspects of experience that are tangential to our research goal. Finally, in relational coding, we mapped some of the categories induced from the data to extant concepts from psychological, phenomenological, and neuroscientific literature.

As mentioned in the Subsection 2.3., throughout the course of the study, data acquisition and analysis were performed in parallel (Flick, 2009). Based on insights gained during the analysis, we inquired about specific questions in more detail. Further, we were able to check whether certain experiential categories we had induced in earlier interviews were valid by asking the participants about them in subsequent interviews (Charmaz, 2004). This means that in parallel to the process of data acquisition, provisional categories were constructed. The validity of these categories could then be checked against subjective reports in subsequent interviews.

We observed a rich continuum of experiences associated with solving the change-detection task. As such, the relational coding yielded a complex taxonomy (Figure 3) of experiential states, spanning five levels of coding (denoted with Roman numerals). On the lowest level of coding (Level I), the categories refer to the smallest degree of abstraction from the raw transcriptions of the interview. As such, they are - for the most part - theoretically unburdened. Moving upwards through the levels of coding, the experiential categories are grouped together based on both their structural (i.e., descriptive) similarities, as well as on working memory literature. For example, we introduced differences in coding that are based on working-memory processes (i.e., target stimuli/encoding, delay/maintenance, and the presentation of the probe stimuli/recall) that a particular aspect of experience is associated with.

\subsubsection{Codebook}

\footnotetext{
${ }^{3}$ Inductive coding is a process through which we ascribe general descriptive tags (usually in the form of words or phrases) to sections of the raw data.
} 
The taxonomy of experiential categories was organized in an annotated codebook. Constructing a codebook (sometimes referred to as the coding manual; Kalinowski et al, 2010) is a standard procedure in qualitative research, and has been productively used in empirical phenomenological studies as well (e.g., Hurlburt \& Heavey, 2006; Kordeš et al., 2019; Schwartzman et al., 2020). Codebook is a text in which each experiential category is described using a) a name; b) a definition; c) logical relationships with other categories (i.e., which categories are superordinate or subordinate to each other; d) representative examples; and e) considerations (in which specific differences between similar categories are explicated and demonstrated with examples). The codebook serves three purposes. First, it provides a way of organizing the large amount of data acquired in the study. Second, it serves as a criterion of validity (i.e., a valid taxonomy of experiential categories yields a logically consistent codebook). Finally, the codebook provides a quick way for independent researchers to familiarize themselves with our coding taxonomy. The codebook is made available in its entirety in SM-C.

\subsubsection{Determining the validity of coding}

To ensure that we have reached conceptual depth (sometimes also referred to as saturation; Saunders et al., 2018), which is the point when we have gathered enough data for constructing a theory, we used the annotated codebook approach (Nelson, 2017). This approach supplements the codebook with a saturation grid (hence annotated codebook). A saturation grid is a tabulation of interviews listed along the horizontal (in our case, we listed individual participants to make the large amount of data tractable) and the codes listed along the vertical. The occurrence of each new code is marked in the appropriate cells. When in several subsequent interviews, no new codes can be induced from the raw data, we can claim that we have reached conceptual depth. Table 1 represents a condensed saturation grid for our study. We have reached saturation after completing the interviews with Participant 14.

Table 1.

Condensed saturation grid.

\begin{tabular}{|l|l|l|l|l|l|l|l|l|l|l|l|l|l|l|l|}
\hline Code/Participant & 1 & 2 & 3 & 4 & 5 & 6 & 7 & 8 & 9 & 10 & 11 & 12 & 13 & 14 & $15-31$ \\
\hline New codes discovered & 22 & 4 & 0 & 8 & 5 & 1 & 2 & 0 & 0 & 2 & 0 & 0 & 0 & 1 & 0 \\
\hline
\end{tabular}


To ensure the validity of our coding process we further took two measures: intercoder verification and consensual validation. The former refers to checking whether two independent coders reached the same types of codes on the same subset of data. To this effect, the codebook was agreed on by the two principal coders, and the entirety of the gathered phenomenal data was subsequently subjected to the same codebook. Consensual validation refers to us checking with the participants themselves whether they agree that our coding process accurately reflects their experience. Consensual validation took the form of additional interviews in which the participants were given the experiential categories and asked to use them to report on their experience according to those categories. In total, five sessions were conducted with participants who were perceived by the researchers as most skilled in observing and reporting on their experience.

\section{Results}

The present section is split into two sections. First, in the Subsection 3.1., we describe the overall experiential styles with which participants approached the visuo-spatial working memory task. These amount to composite descriptions, an otherwise ethnographic style of presentation of qualitative data, whereby telling descriptions are constructed from multiple interviews. In Subsection 3.2., we outline formal categories constructed within this study.

\subsection{Composite descriptions}

In this subsection we present four composite descriptions of the experience of solving a visuo-spatial working memory task. We present four such descriptions, corresponding to four broader accounts of how participants engaged with the visuo-spatial working memory task: a) strategic, analytical performance; b) receptive, meditative performance; c) anxious feeling of being overwhelmed by the task; and d) the task breaking down on account of participants' feelings of despondency.

First, let us examine a case of strategic, highly analytic task-performance.

Participant 05 saw that some parts of the memory stimulus tend towards the same direction. He recognized a visual feeling of density, weight, and compactness at that part of the stimulus. He memorized that part of the stimulus by holding on to a non-verbal awareness of the concept of density. The other half of the stimulus seemed to him more chaotic and dispersed. He failed to analyze it in such a way that it would be describable. So, he made the decision to project the image on screen during the delay period. During the delay period, he focused on the impression of the memory stimulus. Based on these impressions, he imagined two black lines in the mental space in front of him. The black lines corresponded to the dispersed orientation of the chaotic half of the memory stimulus. This was a concretely visual experience, something he saw as floating in the world between himself and the screen. When the test stimulus appeared in front of him, he applied the two rules to it. He checked whether the mental image he had constructed during the delay matches the stimulus on the screen. Then, he looked whether the other half matches the visual feeling of density. He noted a flaw in these rules and after a long time (1935ms) correctly solved the task.

Participant 05 adopted the strategy of looking for what he termed "rules:" Attending to the memory stimulus with the intention of finding the underlying structure of the stimulus and searching for different visual feelings. In the right half of the stimulus, he detected a kind of spatial gradient, a feeling of depth that went into the side of the stimulus, as if the orientations of the object of stimulus curved around the edges of a valley. He assigned the rule depth to this visual feeling. This rule was concretely verbalized in his inner speech. At the same time, he paid 
attention to the other two objects of the stimulus, apparent to him in his peripheral vision. He was aware only of their general direction. These two stimuli were beyond his rulemaking.

Simultaneously, he was also aware of an auditory feeling that was physiologically impossible to replicate; it had a mechanical quality. This sound was related to how Participant 05 scanned the stimulus with his gaze. It seemed to the participant that this sound somehow encoded the orientations of individual objects.

Participant 05 is highly engaged with the task. He employs various strategies to become familiar with different aspects of the stimuli, and subsequently attempts to memorize them. Two aspects of this experiential report are particularly meaningful. First, Participant 05 performed several different experiential gestures to memorize the stimulus (e.g., finding rules, naming them, paying attention to his peripheral vision). Secondly, the clearest aspect of his phenomenology is the attentional disposition of going towards the task, of analytically approaching it and performing several mental operations upon it. It seems that the attentional disposition and mnemonic strategies amount to almost the same aspect of experience even though the former is categorized as a background feeling and the latter as active memorizing.

Explicitly strategic manner of solving the visuo-spatial working memory task, however, is not the only approach participants took. Consider the following example in which Participant 14 had no apparent mnemonic strategy:

Participant 14 experiences the task not as a test or something that is to be performed well, but just as something that happens to appear in her awareness. As the memory stimulus is presented to her, she simply looks at it, allowing the image to naturally appear to her such as it is. In other words, she does not perform any mental gestures upon it. She simply observes the image with a vague, removed sense that - when the test stimulus arrives - the answer will be readily available to her. During the delay period, the participant is not explicitly aware of what the memory stimulus was like. She is aware of a vague, raw feeling that just a moment before, there was something on the screen. As the test stimulus is presented to her, the participant immediately knows whether it is equal to the memory stimulus. She does not perform any actions to become aware of this. This feeling of knowing is not something explicitly experienced. It is simply an absence of doubt or disturbance in which the stimulus gives itself to her as equal.

Further, let us consider some participants who experienced extreme discomfort. Over the course of the validation interview, Participant 22 reported that whenever she was solving the orientation condition, she consistently experienced anxiety. Consider the following report:

"The memory stimulus appeared on the screen, and I immediately realized that I do not have the time to encompass the whole image with my eyes, let alone memorize it. I was moving my eyes around. I was panicking. I was trying to construct a memory, a pattern that would be sensible enough to be memorable. When the test stimulus actually happened, I had no explicit memory representation. There was no sense of how familiar the second stimulus was. So, I guessed the answer." (VR.WM.1.22-01-O-01)

Consider the further report from Participant 01 about the negative feelings incurred by the task:

"It was terrible! I felt really unpleasant because I was fixated on having to get all of them right. I can feel the tension in my body, and I feel stressed. I have a feeling that this is how tasks are supposed to be performed. This is not research. It's an exam. It's something I have to get right. I know I have to get it right. This was constantly in the front of my awareness. And it took a lot of attention for me to be able to solve the task. I couldn't find any reference point that would help me 
remember it. I just waited for [the stimulus] to happen and try to remember its impression somehow. I was never sure about the answer. I always doubted the answer."

(VR.WM.1.01-04-P-01)

The transcript of the interview does not do justice to the participant's distress. In research memos, we noted that her face became flushed, she was visibly uncomfortable, and bordering on tears. Participant 27 similarly reports:

"It seemed to me like it should be fun, but it isn't. Now, it's stressful. I am using only about $20 \%$ to pay attention to it and I kind of don't care about it. I started to think about the task and now it's no longer as fun. And everything began going by so quickly. And so, I started thinking, why should I even bother if everything is going by so quickly. And then I ask myself, what's wrong with me? Am I dumb? And so, I just refuse to answer." (VR.WM.1.27-04-O-02)

These four examples - engaged, analytical performance, relaxed, mindful performance, the experience of stress and being overwhelmed, and finally, the experience of giving up - represent four typical attitudes with which participants experienced their engagement with the task. Each of these attitudes, however, is associated with varying underlying phenomenology. The specific experiential categories associated with them will be discussed in the following section.

\subsection{Codebook}

As mentioned in the Analysis section, we organized the qualitative data in the so-called annotated codebook. The experiential categories in the codebook span five levels of coding, with lower levels representing the smallest degree of abstraction from the raw data (i.e., amount to descriptions of experiential events as reported by the participants), whereas higher levels of coding refer to our attempts at organizing the data according to both descriptive similarities between categories, as well as insights from extant literature. The entire codebook is schematically represented In Figure 3. 


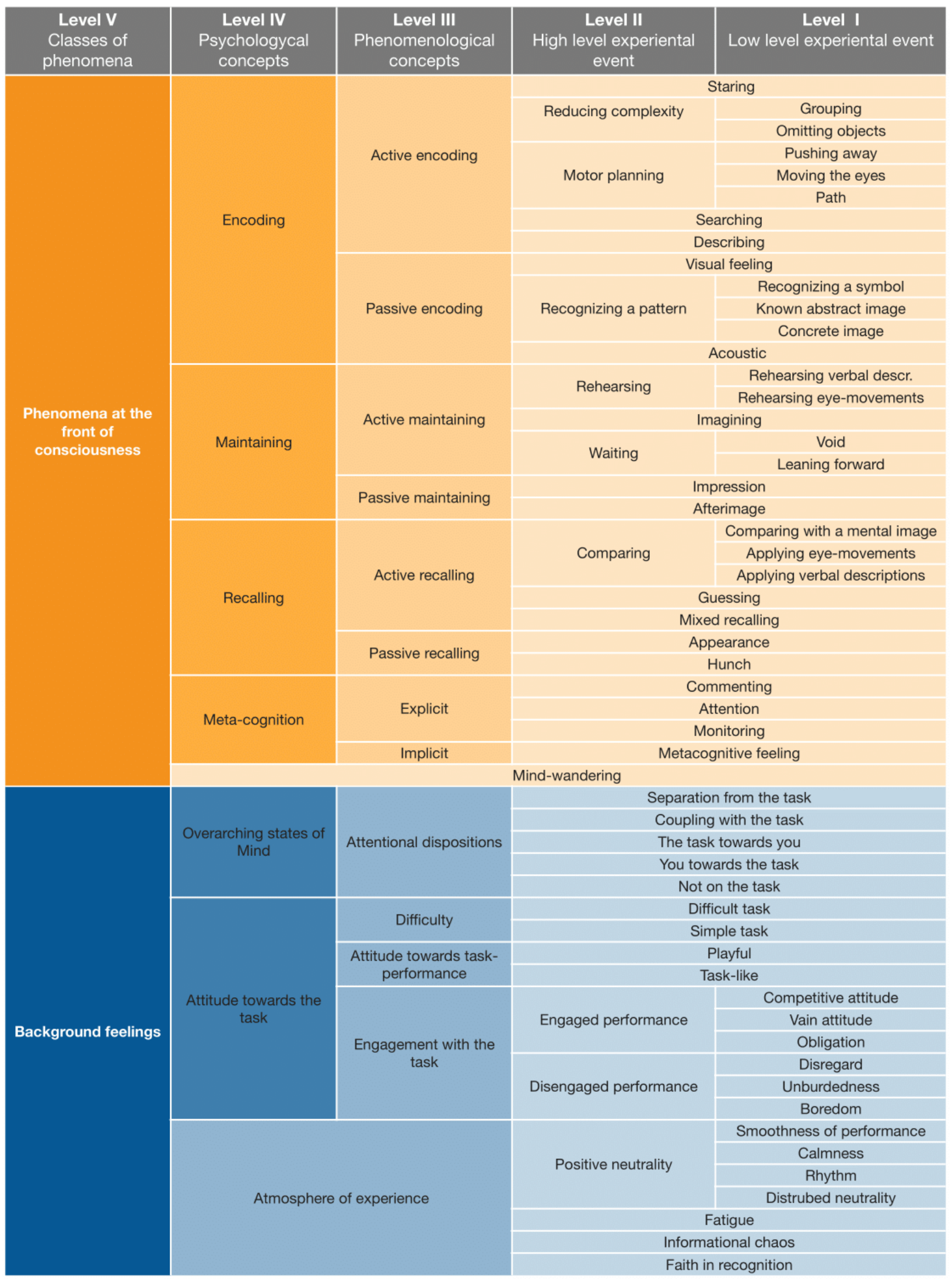


Figure 3. Taxonomy of experiential categories

At level-V of coding - that is, the highest degree of abstraction from the raw data - we differentiate between phenomena at the front of consciousness and background feelings (cf. Colombetti, 2011). Phenomena at the front of consciousness refers to whatever is most present in the forefront of a participant's experience in each moment. These aspects of experience are readily available to conscious reflection even to participants who are not trained in observing and reporting on their experience. Conversely, background feelings describe overall, integrated, and more subtle aspects of experience that were not explicitly brought into the foreground of participants' awareness but nonetheless described how it was to be them when solving the visuo-spatial working memory task.

Phenomena at the front of consciousness consist of five broad categories: encoding, maintaining, recalling, meta-cognition, and mind-wandering. The categories encoding, maintaining, and recalling refer to the experience of the individual stage of the working memory task. Thus, these categories could broadly be referred to as mnemonic strategies, in that, they refer to the experience of attempting to solve the visuo-spatial working memory task. Each of these three categories is further subdivided into active (i.e., an aspect of experience perceived by participants as something they do) and passive (i.e., an aspect of experience perceived by participants as something that happens to them).

\section{Active encoding}

Active encoding consists of a) staring; b) reducing complexity; c) motor planning; d) searching; and describing. Staring refers to the experience of simply gazing at the target stimuli, hoping that you will somehow remember them. Often, the experience of staring is accompanied by the awareness that this strategy is in vain.

Reducing complexity is an experiential category where participants find the target stimuli to be too complex for them to be able to memorize them. For participants to be able to engage with the task, they first must simplify the stimuli for themselves. This is done in two ways, grouping, and omitting. Grouping refers to participants finding a commonality in some subset of stimuli (e.g., based on shape, color, common theme), whereby they can reduce the number of stimuli that have to be remembered. In other words, they remember the groups as a single stimulus. Conversely, omitting is an experiential category where participants - because of the complexity of the stimuli - consciously choose to remember only a subsection of stimuli, hoping that it will be enough for them to be able to recognize whether the probe stimuli are equal to or different from the target stimuli.

Motor planning refers to an experiential category that describes instances in which participants memorize the target stimuli by memorizing how they looked over them with their gaze. This category consists of three closely related but experientially different aspects of experience. Pushing away is the experience whereby individual stimuli feel as being weighed in space and moving across them with one's gaze is subjectively experienced as going against the apparent resistance of the stimuli. Moving the eyes is a strategy of memorizing the target stimuli whereby participants pay attention and remember the feeling in their eye muscles as they look over the stimuli. Finally, path refers to memorizing the trajectory of the center of the gaze (which may or may not be accompanied by the faint, bright line following it).

Searching is an experiential category that describes active encoding whereby participants attempt to discover a pattern in the stimuli, however, they need not actually find the pattern for this strategy to be successful. Finally, describing refers to participants tagging the target stimuli with some form of linguistic description, typically rendered in the form of inner speech.

\section{Passive encoding}


Passive encoding refers to a set of experiences of the target stimuli whereby participants feel that memorization is an aspect of experience that happens to them. Passive encoding consists of three subcategories: a) visual feeling; b) recognizing a pattern; and c) acoustics. Visual feeling is the experience of the overall visual atmosphere of the stimuli (e.g., blue, white, and black stimuli feeling cold).

Recognizing a pattern is an experiential category that describes the experience of some conceptual knowledge to describe the stimuli being immediately available upon perceiving them. Recognizing a pattern consists of three subcategories. First, recognizing a symbol refers to an awareness that there is some symbolic structure that can be readily related to the stimuli (e.g., white, green, and red being memorized as the colors of the Italian flag). Second, known abstract image refers to stimuli being describable through some geometrical shape (e.g., dots in the location condition outlining the shape of a star). Finally, concrete image describes instances where the stimuli elicit a mental image that incorporates some aspect of their appearance (e.g., in location condition, dots arranged in the shape of a rhombus might elicit a mental image of a kite under a blue sky).

The final category of passive encoding is acoustics. This aspect of experience refers to participants being aware of apparent sounds associated either with the appearance of an individual stage of the task or individual stimuli. Occasionally, this imaginary sound component may be sufficient for successful memorization.

\section{Active maintaining}

Active maintaining describes participants willfully attempting to hold the target stimuli in their awareness during the delay period of the visuo-spatial working memory task. Active maintaining consists of three subcategories: a) rehearsing; b) imagining; and c) waiting.

Rehearsing is an experiential category that refers to some aspect of experience being continuously repeated in a participant's awareness for them not to forget the target stimuli. Rehearsing consists of two subcategories: rehearsing a verbal description and rehearsing eye-movement (in which the method of active memorizing of motor planning is rehearsed).

Imagining describes the experience of the target stimuli being recreated in one's imagination using mental imagery (i.e., the target stimuli are maintained in the visual modality).

Finally, active maintaining consists of waiting. Interestingly, waiting was experienced as something that was actively performed by participants. It consists of two categories: void and leaning forward. The former describes an awareness of the absence of mental content. Participants are simply waiting for something to happen. Conversely, leaning forward describes the experience of performing a gesture towards the future moment (e.g., by attempting to anticipate or predict the identity of the probe stimuli).

\section{Passive maintaining}

Passive maintaining is an experiential category that describes instances where the target stimuli remain in participants' awareness seemingly of their own accord. No explicit mental acts are required for these aspects of experience. Passive maintaining consists of two subcategories: a) impression; and b) afterimage.

Impression is an awareness of marked space left behind by the disappearing target stimuli during the delay period. It is a spatial feeling of something having been there. Conversely, afterimage is a visual 
experience in the form of a rapidly deteriorating echo of the target stimuli. Afterimage is typically the opposite (i.e., contrast) color to the stimuli.

\section{Active recalling}

Active recalling is the experience of participants attempting to use an explicit strategy to determine whether the probe stimuli are equal to or different from the target stimuli. Three strategies of active recalling were identified: a) comparing; b) guessing; and c) mixed recalling.

Comparing is an experience in which two explicit experiences at the foreground of one's consciousness are compared one against the other: first, there is some explicitly present memory of the target stimuli, and second, the visual presence of the probe stimuli. Three common experiences of comparing were observed: comparing with a mental image (i.e., a visually present memory of the target stimuli), applying eye-movements, and applying verbal descriptions.

Guessing refers to participants not having an explicitly present answer to the probe. Thus, they opt for picking an answer at random.

Finally, mixed recalling refers to a highly specific experiential dynamics during the probe stimuli. Namely, participants first passively become aware of whether the probe stimuli are equal to or different from the target stimuli (see below for passive recalling). In absence of an explicit strategy, participants feel unsure in their response. Thus, they use an additional explicit strategy to make sure that their initial feeling is correct.

\section{Passive recalling}

Passive recalling is an experiential category that describes instances in which the knowledge about whether the probe stimuli are equal to or different from target stimuli occurs to the participants. Passive recalling contains two subcategories: a) appearance; and b) hunch. The two subcategories differ primarily in their location within one's experiential field, and the level of certainty. Appearance is an experiential category that describes the answer being present immediately in the perception of the probe stimuli. Appearance is accompanied by a sense of absolute certainty that the answer is correct. Second, hunch is a bodily-felt likelihood regarding the answer to the probe stimuli (cf. gut feeling). Unlike appearance, hunch is subject to doubt.

\section{Meta-cognition}

During the performance of the visuo-spatial working memory task, participants commonly reflect on their performance. We coded such experiences as meta-cognition. Subcategories of meta-cognition are divided along the lines of how saliently they are present in participants' awareness: a) explicit; and b) implicit. Three explicit aspects of meta-cognitive phenomenology were observed. First, commenting refers to an ongoing monologue or a dialogue, rendered in inner speech, providing feedback on what participants are doing and how well they are performing at the visuo-spatial working memory task. Second, attention refers to a sense of self-assuredness associated with the knowledge that they are paying close attention to the task. Since they are mindful of the task, the logic goes, it is unlikely that they missed something. Finally, monitoring refers to participants assuming a variety of observational perspectives of their experience of the task, both to make sure that there are no experiences that slip past them, and to be able to report on their experience. 
The only implicit aspect of meta-cognitive phenomenology is meta-cognitive feeling. This experience is closely related to the experience of positive neutrality (described in detail below). Meta-cognitive feeling refers to whether participants suddenly become aware of some disturbance in their field of experience, signifying that they have made a mistake.

\section{Mind-wandering}

The final phenomenon at the front of consciousness refers to mind-wandering. These are instances in which participants' attention moves away from the task towards other objects and/or mental activities, unrelated to the visuo-spatial working memory task, that they construct for themselves.

It is important to emphasize that many phenomena at the front of consciousness occur simultaneously or overlap within a single experiential episode. Consider Table 2 for an example of how the categories presented here were extracted from raw data during the coding process:

\begin{tabular}{|c|c|c|c|}
\hline Raw data & $\begin{array}{l}\text { Inductive } \\
\text { coding }\end{array}$ & $\begin{array}{l}\text { Relational coding } \\
\text { (low-level coding) }\end{array}$ & $\begin{array}{l}\text { Relational coding } \\
\text { (high-level coding) }\end{array}$ \\
\hline $\begin{array}{l}\text { I realized that the [stimuli] had the } \\
\text { form of a kite. This kite aroused } \\
\text { certain emotions in me. They } \\
\text { weren't too intense. Without these } \\
\text { emotions, I wouldn't be able to } \\
\text { come up with this association. I saw } \\
\text { a blue sky and an orange kite. I } \\
\text { concretely saw the orange, whereas } \\
\text { the blue was more in the } \\
\text { background. I saw the kite on the } \\
\text { screen. The screen beyond it had } \\
\text { depth. So, the kite was located in } \\
\text { this space. However, this depth } \\
\text { didn't add any meaning. It doesn't } \\
\text { help in any way. (S14-04-P-02) }\end{array}$ & $\begin{array}{l}\text { A concrete } \\
\text { image } \\
\text { Affect } \\
\text { Affect - weak } \\
\text { Affect } \\
\text { A concrete } \\
\text { image } \\
\text { A concrete } \\
\text { image } \\
\text { Projected image } \\
\text { Depth } \\
\text { Projected } \\
\text { image, depth } \\
\text { Not useful } \\
\text { Not useful }\end{array}$ & $\begin{array}{l}\text { Concrete image } \\
\text { Projection } \\
\text { Epiphenomenal }\end{array}$ & $\begin{array}{l}\text { Recognizing a pattern } \\
\text { Imagining } \\
\text { Appearance }\end{array}$ \\
\hline
\end{tabular}

Table 2.

Phenomena at the front of consciousness are of particular interest to the theoretical construct of working memory, specifically, what kind of cognitive strategies, as accessible to conscious reflection, participants deploy to successfully solve the task. A detailed description of the categories will be presented elsewhere. In the remainder of this paper, we pay particular attention to the second Level-V experiential category: background feelings.

\section{Background feelings}


During data acquisition and analysis, we observed a huge variability in non-strategic experiences during the task-performance. While aspects of experience related to strategic performance of a visuo-spatial working memory task (outlined above) were - in a manner of speaking - artificial (being tied to specific stages of the working memory task), we observed an aspect of experience that provides a much more holistic description of how it feels to solve a visuo-spatial working memory task. These aspects of experience represent the second level-V category: Background feelings. Background feelings constitute aspects of experience that are not placed in the focus of participants' awareness, but nonetheless play a major role in the description of how it is to be someone in each moment. They are represented by several subtle attentional modulations, and bodily feelings.

Notably, while phenomena at the front of consciousness are readily accessible even to participants who are not trained in observing one's experience, background feelings are not as easily apparent in conscious reflection. Background feelings include the following level IV subcategories: a) attentional dispositions, b) atmosphere of experience, and c) attitude towards the task. These aspects of experience will be explored in detail in subsequent sections.

\subsection{Attentional dispositions}

Attentional dispositions describe different attitudes we can take within our attention towards a specific object of our awareness. In turn, this attitude influences how we experience the object itself. In this study, we observed five different experiential categories that we consider to constitute attentional dispositions we may take towards a visual-spatial working memory task: separation from the task, coupling with the task, you towards the task, the task towards you, and not on the task. Separation from the task refers to an attitude of distance towards the change-detection task. Rather than being experienced as something that the participant has a causal effect over, it is seen more as a video being passively observed. As Participant 27 reports:

"I wasn't really with the task. I was solving it and it was important to me to solve it well, but I was also really aware of the room and you sitting behind me [...] What happened was that there was this sense of whole that was left over from the previous example, and then I didn't know whether I'm comparing it to this example or the one that came before. I was thinking about that." (VR.WM.1.27-04-O-01)

Conversely, coupling with the task is an experiential category that describes a spatially felt connection between the participant and the task at hand. This connection can be implicit and can appear simply as a loss of awareness of the research setting, the researcher, and the environment. Conversely, it can be experienced very apparently, as a sense of an enclosed, private space between the participant and the psychological task. Consider the following:

"It is a kind of bubble. My attention is concentrated here at the front, at the task. This bubble is essentially a very pleasant kind of attention. I really like this focus. And my surroundings disappear. And I'm not aware of you either. There wasn't even an awareness of where I'm sitting. All that existed was this task. And I felt warm about that. This warmth was for me and for the way I do the task. It wasn't warm like the sun, but like a warmth in my thoughts."

(VR.WM.1.24-01-P-02)

Alternatively, participants can approach the task by performing several mental gestures upon it. This experience may be so strong that it appears as a sense of direction moving from the participant towards the task. Consider the following example where the performance of mental gestures is explicitly reported on: 
"The task seemed more difficult than before. Before, it was enough for me to just have the impression of the image, whereas now I had to keep more of my focus on the task. I had to push away the things unrelated to the task. I performed movements with my gate from object to object. I was jumping across them." (VR.WM.1.10-01-C-06)

Participants may also assume the attentional disposition of the task towards you. This experiential category refers to the participants appreciating the stimuli such as they are, without performing mental gestures upon it; that is, the category constitutes a receptive attitude towards the task. Consider the following report:

"I just let the dots happen [...] I trusted myself that I will be able to know whether the next stimulus is correct or not. [...] It was as if I shut the task down. I was looking at the [fixation cross] and it was a little bit like meditating. I didn't have to think of the dots or anything." (VR.WM.1.15-02-P-04)

The final attentional disposition that we have constructed within this study is not on the task, which refers to situations where participants attended to something other than the visual-spatial working memory task. This attentional disposition, however, is quite rare, as it was difficult to come across cases where the task did not in any way enter participants' awareness without the research context breaking down (i.e., without them stopping to solve the task).

\subsection{Attitude towards the task}

The second subcategory of background feelings are attitudes towards the task. These are background feelings within which the participants implicitly interpret the experimental paradigm in one way or another. In this context, we use the term "interpretation" to refer to what the participants make of the whole research setting. Attitudes towards the task contain three major ways of interpreting the change-detection task: a) difficulty, b) attitude towards task-performance, and c) engagement with the task.

Difficulty is an attitude towards the task in which the participants interpret the task either as easy or difficult. Importantly, it is apparent that the difficulty of the task is not a property of an observer-independent, objective world, but it amounts to an attitude that participants take. The change-detection task can be appraised as either:

\section{1) Difficult;}

2) Simple.

Engagement with the task is an experiential category that describes the level of enthusiasm and zeal with which the participants approach it. In other words, it is a question of whether the participants approach the task as if it is important and something they must do well, or whether they approach it as something irrelevant, something that is simply there. Engagement with the task can be subdivided into engaged performance (where it is important to participants that they do well on the task), and disengaged performance (where the task is no longer at the center of their awareness). The former contains the following subcategories:

1) Competitive attitude, exemplified by the following:

"I am doing well. It is important to me that I am doing well. I don't know. It feels like I am competing with somebody. Maybe I am competing with myself or maybe I am competing with the computer. In any case, it's important that I'm doing well." (VR.WM.1.24-01-C-01) 
2) Vain attitude, exemplified by the following:

"I have to do this. It's not as if there are some positive or negative consequences if I succeed or fail. It's a challenge. I accepted the challenge. So, now it becomes the question of whether my capabilities - mental capabilities are up to par. Whether I can do it. I'm curious about that. I'm curious about whether I can do it." (VR.WM.1.27-04-O-01)

3) Obligation, exemplified by the following:

"I woke up from snoozing. It felt uncomfortable. As if I knew I can't fall asleep, and yet, I am still sleeping. There was an imperative to me solving the task. Even though I wasn't actually solving it, I experienced some sort of I must. It's a kind of presence. I didn't create it. Well, maybe at some point, I maintained it. First it came from the outside and then I continued it. (VR.WM.1.25-03-O-03)

Disengaged performance contains the following subcategories:

1) Disregard, exemplified by the following:

"I couldn't even see the screen. I turned off. I wasn't present. I couldn't see the objects. I could still feel some sort of rhythm, but this wasn't enough for me to be able to solve the task [...] The task is completely beyond my experience. I remember noticing that at some point the [fixation cross] turns dark, and that was the only time I was paying attention to the task for even a little bit." (VR.WM.1.25-02-P-05)

1) Unburdedness, exemplified by the following:

"I was thinking about how nobody's even going to look at these results. And that made me much more relaxed. I was no longer thinking about whether I am solving it right or wrong. This feeling was there from the very beginning. It wasn't really bodily. It was just a thought."

(VR.WM.1.25-02-O-01)

2) Boredom, exemplified by the following:

"I kind of got sick of it. My attention started to wane. I experienced this tiredness in two parts.

One was this end to trying my best. The other was wandering of my attention. I still gave informed answers, only now, there was a greater sense of guessing because I no longer tried to maintain representations during the pause." (VR.WM.1.10-01-O-02)

\subsection{The atmosphere of experience}

The final subcategory of background feelings is the atmosphere of experience. It is a background feeling that describes the frame of the participant's experience. It refers to those aspects of experience that do not carry with them a specific content, but rather color whatever is at the center of the participant's experience. It contains four subcategories: a) Positive neutrality, b) fatigue, c) informational chaos, and d) faith in recognition. Fatigue is an experiential category that is to be understood in the trivial sense of the word - the experience of being tired. Consider the following report, demonstrating the atmospheric nature of fatigue:

"I suddenly became aware of the light. Of how strong it is. I am actually still aware of that now. It was as if the light burnt me a little bit. This is why the task seemed more difficult. I think I had to 
pay much more attention to it. The task was suddenly very far away. It was as if I did not have enough focus." (VR.WM.1.31-02-P-02)

Faith in recognition refers to an experience where despite not having an explicit mnemonic strategy, the participants feel that they will be able to successfully solve the change-detection task:

"I know I can do it. It's just that I don't know how exactly I know. When I look at the memory stimulus, it just seems like I will be able to remember it." (VR.WM.1.07-01-C-02)

Informational chaos is a particular atmosphere of experience where the visual-spatial working memory task seems so complex as to be impossible to solve. It seems impossible to memorize the memory stimuli on account of how complex they seem. Participants commonly experience this complexity as the absence of any stable point at which to direct their attention to start memorizing the stimulus. It is an exceedingly unpleasant experience, and it is accompanied by feelings of anxiety and fear (Kordeš, 2019).

One of the key aspects of informational chaos is the experience of the lack of stability, that is, the sense that there is no part of the visual experience that the participants could anchor their attention to in order to start remembering. Participant 25 reports:

"I ran out of focus, but not because I was wrong with the previous one. It was simply no longer clear what I have to do. I don't understand what is happening on the screen. I don't know what to do. It was really, really uncomfortable. This panic was much worse than if I was just wrong. I almost started crying. I felt totally powerless. I felt this tension that was rising from my chest to my clavicles. I felt that I have an increased heart rate. I didn't know what to do. There was a confusion in my mind. I didn't know what to focus on. There was a lot of pondering, but actually, I didn't really have any thoughts. It was all mostly bodily experience. I couldn't stop it. I couldn't make a decision about what to do. This panic was the only thing I was really experiencing. I wasn't solving the task. I was just randomly clicking. Because I knew I had to. And there was this idea of a record of everything that I don't click on it." (VR.WM.1.25-01-O-02)

She goes on:

"I began thinking about how I don't like the exercise. The tails of the objects bothered me. They caused these bad feelings inside of me. These were more mental than bodily. It wasn't as if I was in pain or under stress. It was more of a preference. As if I didn't have these tails on the objects." (VR.WM.1.25-03-O-02)

Importantly, informational chaos is associated with a particular attitude towards the task, namely the sense of obligation. As Participant 22 reports:

"I had a feeling of a total lack of control. I knew that I could not answer these questions. I randomly clicked. And when I finally gave into it, I had no thoughts about it, even though it encompassed the better part of my experience." (VR.WM.1.22-01-0-01)

The performative dimension of informational chaos is apparent: the participants are no longer solving the task, but are rather clicking at random, because of an experience of obligation, because they must. Rather than asking for the study to end, they go on performing the performance of a visual spatial working memory task.

Importantly, however, in $69 \%$ of cases in which informational chaos was observed $(\mathrm{N}=16, \mathrm{C}=11)$, the participants were still able to provide a correct response. In conclusion, our participants - while experiencing informational chaos, an aspect of experience associated with the complete collapse of the research setting in an individual's experience - still performed at above-chance rate. Further, this rate of 
successful answers is comparable with the threshold that visual-spatial working memory research sets as the criterion for the upper limit of working memory capacity (cf. Luck \& Vogel, 1997)

Positive neutrality is an atmosphere of experience in which the participants are aware of the absence of any kind of disturbance. It is the sense of the smooth running of events. This smoothness; however, is not experienced positively in and of itself: it is a noticeably neutral aspect of experience. Because the participants are aware of nothing being wrong, this neutrality is experienced as something good, as something mildly positive. Positive neutrality may be brought into one's awareness four interrelated and connected ways:

1) Smoothness of performance;

2) Calmness;

3) Rhythm;

Smoothness of performance is an atmosphere of experience in which the participants are aware of positive neutrality at their own task performance. The task - and its performance - run smoothly, without any interruptions. This smoothness in and of itself is not pleasant. The absence of disturbances is the aspect of experience that is perceived as positive. Consider the following:

"Everything was related to the rhythm. It created this flow. This rhythm was silent, discrete, a staccato. I moved my eyes according to where I was expecting the dot to appear. When it didn't appear, I got a sense of dissonance. It went outside of the rhythm. It's a very mental experience, yes, but it is not reasoning. It wasn't as if I said 'Aha, it went out of rhythm, therefore, it does not fit.' It was a more subtle feeling. Like an aha moment. The dot was not here, so it must be there. When the dot was in an unexpected place, the rhythm broke down. And so did any image that I had in my mind." (VR.WM.1.19-02-P-02)

The experience of rhythm is, by and large, tied to the appearance of stimuli themselves. This experiential category refers to an embodied (and sometimes even auditory, when accompanied by the category acoustic component) element of experience. Participants report that rhythm structures their time and that task-performance in consequence becomes more relaxed and predictable. Rhythm can be embodied and is commonly tied to the feeling of heart rate. In a similar manner to the smoothness of performance, rhythm can break down in the case of a mistake or a disturbance in an experiential field. This leads to the tempo stopping. Insofar as the disturbance does not occur, the rhythm encompasses the final key press as well: "A rhythm of performing the task established itself. At the end, I answer completely automatically. If there's a mismatch, this way of solving the task stops." (VR.WM.1.17-04-O-02)

Smoothness of performance is an experience that is structurally like rhythm from the point of view that once it is present, it appears as the default state of the experiential landscape. Both aspects of experience may be cut short by a disturbance. The difference is that smoothness of performance is, by and large, unconscious. Rhythm, on the other hand, clearly constitutes a presence.

Consider the following example:

"I said to myself, 'one, two, three, four.' This happened in the same rhythm as the dots appeared in. these verbalizations were sounds that happened in my own voice. The rhythm helped me concentrate. It gave structure to my sense of time. It wasn't intended to be a dancing rhythm but it reminded me a lot about dance where you have to count [...] I'm the author of these words. I willfully produce them. When they first appeared, I didn't willfully create them. It wasn't my strategy at first. But then I noticed that I'm doing it and that it feels good. What felt good was the structure. I latched on to that and started actively doing it." (VR.WM.1.04-02-P-01) 
Finally, calmness is an atmosphere of experience whereby the participants feel pleasant in the absence of disturbances. Calmness itself has no object. It describes the general way of existing in a particular moment.

\section{Discussion}

In the present study, we investigated the experience of solving a visuo-spatial working memory task. We used the change-detection task, a standard instrument for studying working memory, and gathered first-person data with a combination of experience sampling and an in-depth phenomenological interview. This resulted in a large array of data depicting the experience of participants solving the visuo-spatial working memory task. During the study, we observed that the experiential landscapes associated with visuo-spatial working memory task-performance are extremely rich, both in terms of mental acts that participants use to attempt to "solve" the task, as well as in terms of depth of experience; that is, overall experiential states accompanying (and often probably determining) the task-performance.

Our qualitative analysis separated the gathered phenomenal data into two major classes of experience. First, there is an experiential dimension, coded as phenomena at the front of consciousness. This category describes aspects of experience that occupy the center of participants' awareness and are readily accessible to consciousness reflection even in absence of training in how to observe and report on experience. Phenomena at the front of consciousness consist of various strategies of solving the visuo-spatial working memory task, together with meta-cognitive experiences, and mind-wandering.

Phenomena at the front of consciousness represent a methodological bridge between empirical phenomenology and experimental cognitive psychology. These aspects of experience are the easiest to detect, even for participants who are not trained in observing and reporting on their experience. At the same time, phenomena at the front of consciousness may be productively used within experimental cognitive psychology and provide a phenomenological understanding of working memory by accounting for, for example, different cognitive strategies used to successfully solve the same task (cf. Mogensen \& Overgaard, 2018).

Second, during the analysis, an experiential dimension that is harder to access in conscious reflection was consistently detected. We coded this experiential dimension as background feelings. Most of the present article, including the discussion, is dedicated to this experiential dimension of background feelings. The study of background feelings poses significant methodological challenges, but - we suspect - could play an important role in understanding and interpreting the results of visuo-spatial working memory task-performance, as well as working memory more broadly.

In Subsection 3.1., we illustrated the wealth of experiential landscapes through composite ethnographic descriptions of experiences from a handful of participants. We noticed a range of attempts at contending with the task, as well as a host of personal attitudes towards the task. Some of these aspects of experience can rightly be considered strategies of task-performance, whereas others amount to trying to reduce stress elicited by the experimental setting. Further, it is commonplace that participants' attention during task-performance is not consistently experienced as on-task, even though behaviorally, the protocol appears to be nominal. Moreover, sometimes, performance is more successful when participants are not explicitly engaging with the task rather than when they experience intense dealing with the task. Attempting to make sense of experiential data such as this, confronts us with an interesting question: is 
the presence of a conscious component (i.e., conscious dealing with the task) necessary to deem a study using a psychological task as valid?

\subsection{Comparison to existing phenomenological ideas}

The presentation of results in this paper was primarily preoccupied with background feelings. Until recently, this kind of experience was almost entirely unmentioned within psychological literature (although see Tsuchiya \& Koch, 2016). Yet, we believe that understanding this aspect of experience may be essential (perhaps even more so than understanding the strategies participants use to solve a visuo-spatial working memory task) to understand task-performance. Our findings suggest that aspects of experience associated with a strategic attitude during visuo-spatial-working-memory-task-performance, while frequent and easily detectable, are not the most salient aspect of awareness. Rather, participants experience comprehensive states, which may include a strategy, however, it is also possible that they do not.

By way of example, let us examine positive neutrality, one of the background feelings we identified in this study. Positive neutrality refers to a subtle awareness of an absence of change. Participants report solving the visuo-spatial working memory task guided by the awareness of an absence of disturbances (e.g., wrong answers). This lack of disturbances itself is felt as neutral. However, since - in the context of visuo-spatial working memory task performance - it signifies that the participants have not detected any blunders, it is appraised as slightly positive. In such cases, positive neutrality also reflects the repeating, rhythmic pattern of trials occurring at a predictable rate. Only when something goes wrong (the pace at which they are scanning the stimuli, wrong answer, end of task, etc.) is this positive neutrality disturbed.

These experiential dynamics mirrors the notion of surprise in the so-called free energy principle theories of cognition (Friston, 2009; Friston, 2010). According to this theory, living organisms work to minimize their internal disorder (entropy). They create a barrier between themselves and the outside world, wherein inside of this boundary, they maintain their own homeostasis (Kirchoff et al., 2018; Demekas et al., 2020). A surprising event in this framework, is an event that was not predicted by the organism (Schwartenbeck et al., 2013). When applied to cognition, free energy principle is related to predictive processing, the notion that to minimize the computational complexity of incoming sensory information, organisms predict the likeliest situation and then only process surprising stimuli (i.e., stimuli that they were unable to predict) (Seth, Suzuki \& Critchley, 2012; Seth, 2014, Clark, 2016).

The properties of some of the background feelings (e.g., the feeling of positive neutrality) are remarkably like phenomenological properties associated with or allowing for the experience of surprise (Bitbol, 2019). Integrating these ideas with our own, we posit that positive neutrality is the stable state of successful prediction. When positive neutrality is disturbed, the experiencing individual is prompted to acknowledge change. This, however, is possible only in the case when some foreground activity (e.g., concerning oneself with strategic task-performance) has not clouded participant's access to the background feeling of positive neutrality.

Second, the phenomenon of attentional dispositions has been commonly reported in empirical phenomenological literature. Originally, it was reported in the study on how we experience attending to sound. Attentional dispositions refer to the changes in our experience of the source of sound when we attend to it with different attitudes (e.g., as a physical sensation on our ears, as a location in space, as an 
acoustic experience with sound, pitch, and timbre)(Petitmengin et al., 2009). The findings were corroborated in a study on the experience of meditation (Kordeš et al., 2019). Specifically, participants reported experiencing pain (e.g., in their legs or back when meditating). Pain could then be attended to with different attitudes, which, in turn, altered the intensity of noxious sensations. In the study presented here, attentional dispositions refer to different ways of relating to the task (i.e., the stimuli are approached, the stimuli are allowed to emerge to participants' consciousness, a synchrony is felt with the computer on which the task is displayed, or, there is a distance between the computer and the participants). These descriptions bear resemblance to what Oblak, Boyadzhieva and Bon (2021) refer to as affective resonance, a property of objects present in one's environment that they can jointly form higher-order systems (see also Thompson, 2007; Kyselo \& Tschacher, 2014). This description corresponds to the disposition of coupling with the task, where participants report feeling that the task-performance is not something that they do themselves, but they do it in concert with the computer. Conversely, the disposition separation from the task is descriptively similar to the phenomenological notion of detunement. Detunement refers to an inability of accessing socially shared space in depression (Fuchs, 2005, 2007), schizophrenia (Silverstein, Demmin \& Škodlar, 2016; Krueger \& Aiken, 2016) and anxiety (Trigg, 2017). It may be that part of why individuals with specific psychopathologies underperform on working memory tasks (Rose \& Ebmeier, 2006; Christopher \& MacDonald, 2010; Forbes et al., 2009; Moran, 2016) is because phenomenologically, the task are not salient enough in their awareness for them to be able to attentionally engage with them.

\subsection{Towards a neurophenomenology of working memory}

Positive neutrality and attentional dispositions are not the only example of an experiential category where we observed background feelings playing an important (and perhaps essential) role in task-performance. The entire group of experiential categories we coded as the atmosphere of experience was - according to subjective estimates of participants - important when engaging with the visuo-spatial working memory task. Our results seem to coincide with some recent ideas in cognitive science indicating the importance of understanding the general, background atmospheres. One of such ideas is the theory of overarching states of mind (Herz et al., 2020). It proposes that, on the level of neural dynamics, we can observe integrated states that coordinate all other aspects of cognition, such as perception, emotions, thinking, as well as embodied behavior. It is not unreasonable to hypothesize that such overarching states of mind can be detected on the personal level of description as a specific class of background feelings. ${ }^{4}$

The acquired first-person data allow us to put forward a conjecture. It may be that the aspects of experience detected in this study (and coded as background feelings) determine our behavior to a greater extent than the readily accessible phenomena at the forefront of consciousness. It may be that a larger part of visuo-spatial working memory task-performance occurs below the threshold of conscious awareness (cf. Soto et al., 2011; Soto \& Silvanto, 2014; Dutta et al., 2014). Explicit, conscious attempts at taking control over the task-performance (i.e., assuming a strategic attitude) may therefore only serve to interfere

\footnotetext{
${ }^{4}$ Another neuroimaging study that indicates that points towards the same idea is a meta-analysis done by Hawco and colleagues (2021), clustering neuroimaging data from different types of psychological tasks. They uncovered clusters of neural activity that remain invariant across different psychological tasks. One of the possible interpretations of these clusters is that neural activation is meaningfully associated with cognitive styles.
} 
with task-performance. In other words, attempt at explicit performance stops subconscious task-performance (cf. Lyubomirsky et al., 1999)

However, when talking about a large part of task-performance taking place below the threshold of conscious awareness, we are referring to an everyday, and, for the most part, inaccurate awareness. Is it possible that a more precise insight into the content of consciousness allows this threshold to be raised? What if a more detailed reflection can be used to detect traces of cognitive processes taking part in visuo-spatial working memory task-performance? We believe that this is the case and that these traces can be observed, but only by participants, skilled enough to be able to access background feelings (cf. Petitmengin, Navarro \& Le Van Quyen, 2007). Thus, it is essential for the validity of studies attempting to provide an in-depth account of first-person data to use participants who are skilled and trained in observing and reporting on their experience (cf. Miyahara et al., 2020).

We hope to have shown that aspects of experience that are not so easily identifiable form a significant part of an individual's experiential landscape. Detection of such aspects of experience requires participants who, at the very least, are interested in exploring their own experience, but preferably are also trained in observing and reporting their experience.

Our results correspond to various observations by many within the field of first-person research (Hurlburt, 2011, Kordeš, 2016, Petitmengin, 2017), thereby indicating the necessity of re-examining the traditional roles of researcher and participant (cf. Orne, 1962) in the domain of researching experience. Since the participant is the only person with the access to experience under investigation, then, her process or reflection constitutes the principal instrument of inquiry. If this instrument is inadequate, then, no subsequent step (e.g., interview, analysis, etc.), no matter how refined, can improve the validity of a study. Thus, it is necessary to consider the people whose experience we are investigating not as passive subjects from whom data can be objectively extracted, but rather as equal partners in the research process - as co-researchers (cf. Kordeš \& Klauser, 2016).

The study presented in this paper only partially succeeded at recruiting participants with enough interest and skills in observing and reporting on their experience. They were sufficient to clearly point to the entire "background" aspect of experiential landscape associated with visuo-spatial working memory task-performance. However, too few such participants were recruited for us to be able to gather enough data on background feelings to be able to draw statistical conclusions about their role in visuo-spatial working memory task-performance. Thus, our earlier claims about the essential role of this aspect of experience for the understanding of visuo-spatial working memory task-performance amounts to a working hypothesis.

In future research, we aim at replicating the study presented in this paper, however, recruiting only participants trained in observing and reporting on their experience. Additionally, we hold that using trained participants may be beneficial for future fMRI and neurophenomenological studies (cf. Fernyhough et al., 2018; Lutz et al., 2002). As demonstrated by Hurlburt et al. (2016), validity of studies assuming that the experimental paradigm constructs the phenomenon of inquiry, without verifying it on the level of experience, is questionable (Hurlburt, 2011).

Further, detailed reports from trained participants could be subjected to dimensionality-reduction; that is, constructing experimental categories that are intuitively understandable and therefore usable as 
independent variables in a neuroimaging research design, will be conducted on a small sample of individuals who are highly trained in observing and reporting their experience. Such dimensionality-reduction would make the taxonomy presented in this study pragmatically useful as an instrument to report on one's experience within a neuroimaging study. This means that the categories will be well-defined for the participants, making them easy-to-use within an EEG or an fMRI setup (so-called front-loaded phenomenology (Gallagher \& Sørensen, 2006).

In line with Hurlburt and colleague's (2016) findings, some insights, associated with background feelings, indicate that the experience of visuo-spatial working memory task-performance are quite removed from day-to-day experiences. Participant 22, for example, reports feelings (coded as informational chaos) that are familiar to her only from other times when she was solving similar psychological tasks:

I remember solving a similar task for [one of the professors]. I was stuck in some lab, I was a little bit afraid, and on top of that, there was this bombardment of stimuli that was totally overwhelming. I really don't recognize this feeling from situations other than stuff like this. (VR.WM.1.22-01-O-01)

Gathered phenomenal data raise questions about the validity of studies operating under the assumption that psychological tasks elicit only the target phenomenon without checking participant's experience of said task. Our study has not gathered sufficient data to conclusively reach this conclusion. Additional investigations of experience underlying performance of visuo-spatial working memory tasks, as well as more ecological (e.g., ethnographic) research of day-to-day experience associated with visuo-spatial working memory are needed. Thus, this study points to some as of yet unanswered questions in the broader field of working memory research.

\section{Conclusion}

In this paper, we presented an empirical phenomenological study of how it feels to solve a visuo-spatial working memory task (specifically, the change-detection task). Using a combination of experience sampling and an in-depth interview we gathered a large amount of experiential data. The data were analyzed according to the principles of constructivist grounded theory. The resulting experiential data demonstrate the wealth of different experiences associated with solving a working memory task. Some of them are easy-to-detect and could be productively used to further approaches within experimental cognitive psychology. Others amount to overarching, integrated states of consciousness within a given moment. These require further neurophenomenological investigation, as well as reconsidering the social dynamics between researchers and participants. Gathered phenomenal data raise questions about the validity of studies operating under the assumption that psychological tasks elicit only the target phenomenon without checking participant's experience of said task.

\section{Funding}

\section{Acknowledgements}

The authors wish to thank Ema Demšar for her help with conducting the study, and Jaya Caporusso, Asena Boyadzhieva, and Maša Rebernik for their help with preparing the manuscript. 


\section{Authors' Note}

\section{Author contribution}

(Using CRediT Taxonomy, http://www.cell.com/pb/assets/raw/shared/guidelines/CRediT-taxonomy.pdf).

Conceptualization, G.R., A.S.O and U.K.; Methodology, A.O, G.R., A.S.O and U.K.; Software, G.R and A.S.O; Formal analysis, A.O. and U.K.; Investigation, A.O.; Writing - original draft, A.O.; Writing review \& editing, A.O, G.R., A.S.O and U.K.; Visualization, A.O. and A.S.O; Project administration, A.O.; Supervision, U.K. and G.R.; Funding acquisition, G.R. and U.K.

\section{References}

Bitbol, M. (2019) "Neurophenomenology of surprise: Sparks of awakening," in Surprise at the intersection of phenomenology and linguistics, ed. N. Depraz, and A. Celle (Amsterdam: John Benjamins), 10-21.

Petitmengin, C., Bitbol, M., Nissou, J., Pachoud, B., Curallucci H., Cermolacce M., and Vion-Dury, J. (2009). Listening from within. Journal of Consciousness Studies, 16:10-12, 252-284.

Oblak, A., Boyadzhieva, A., and Bon, J. (2021). Phenomenological properties of perceptual presence: A constructivist grounded theory approach. Constructivist Foundations 16:3, 295-308.

Thompson, E. (2007). Mind in Life: Biology, Phenomenology, and the Sciences of the Mind. Cambridge: Harvard University Press.

Kyselo, M. and Tschacher, W. (2014). An enactive and dynamical systems theory account of dyadic relationships. Frontiers in Psychology 5:452. doi: 10.3389/fpsyg.2014.00452

Trigg, D. (2017). Topophobia: A phenomenology of anxiety. London: Bloomsbury.

Silverstein, M. S., Demmin, D., and Škodlar, B. (2017). Space and Objects: On the Phenomenology and Cognitive Neuroscience of Anomalous Perception in Schizophrenia (Ancillary Article to EAWE Domain 1). Psychopathology, doi: $10.1159 / 000452493$

Krueger, J., and Aiken, A. T. (2016). "Losing social space: Phenomenological disruptions of spatiality and embodiment in Moebius Syndrome and Schizophrenia," in Phenomenology and Science, ed. J. Reynolds and R. Sebold (London: Palgrave Macmillan), 121-139.

Rose, E. J., and Ebmeier, K. P. (2006) Pattern of impaired working memory during major depression. Journal of Affective Disorders, 2:3,149-61. 
Christopher, G., and MacDonald, J. (2005). The impact of clinical depression on working memory. Cognitive neuropsychiatry, 10:5, 379-399.

Forbes, N. F., Carrick, L. A., McIntosh, A. M., and Lawrie, S. M. (2009). Working memory in schizophrenia: a meta-analysis. Psychological medicine, 39:6, 889-905.

Moran, T. P. (2016). Anxiety and working memory capacity: A meta-analysis and narrative review. Psychological Bulletin, 142:8, 831-864.

Herz, N., Baror, S., and Bar, M. (2020). Overarching States of Mind. Trends in cognitive sciences, 24:3, 184-199. doi: 10.1016/j.tics.2019.12.015

Dutta A., Shah, K., Silvanto, J., and Soto, D. (2014). Neural basis of non-conscious visual working memory. NeuroImage, 91:1, 336-343.

Soto, D., Mäntylä, T., and Silvanto, J. (2011). Working memory without consciousness. Current Biology, 21:1, R912-3. doi: 10.1016/j.cub.2011.09.049.

Soto, D., and Silvanto, J. (2014). Reappraising the relationship between working memory and conscious awareness. Trends in Cognitive Sciences, 18:10, 520-525.

Lyubomirsky, S., Tucker, K.L., Caldwell, N.D., and Berg, K. (1999). Why ruminators are poor problem solvers: Clues from the phenomenology of dysphoric rumination. Journal of Personality and Social Psychology, 77:1, 1041-1060.

Hawco, C., Dickie, E. W., Jacobs, G., Daskalakis, Z. J., and Voineskos, A. N. (2021). Moving beyond the mean: Subgroups and dimensions of brain activity and cognitive performance across domains.

NeuroImage, 231, 117823. doi: 10.1016/j.neuroimage.2021.117823

Tsuchiya, N., and Koch, C. (2016). "The Relationship Between Consciousness and Top-Down Attention." In The Neurology of Consciousness: Cognitive Neuroscience and Neuropathology, ed. S. Laureys, O. Gosseries, and G. Tononi, (San Diego, CA: Academic Press), 71-91.

Friston, K. (2010). The free-energy principle: a unified brain theory? Nature Reviews Neuroscience, 11:2, 127-138.

Friston, K. (2009). The free-energy principle: a rough guide to the brain? Trends in cognitive sciences 13:7, 293-301.

Kirchhoff, M., Parr, T., Palacios, E., Friston, K., and Kiverstein, J. (2018). The Markov blankets of life: Autonomy, active inference and the free energy principle. Journal of The Royal Society Interface, 15:138, 20170792. doi: 10.1098/rsif.2017.0792.

Demekas, D., Parr, T., and Friston, K. J. (2020). An investigation of the free energy principle for emotion recognition. Frontiers in Computational Neuroscience, 14:30. doi: 10.3389/fncom.2020.00030 
Schwartenbeck, P., Fitzgerald, T., Raymond, J. D., and Friston, K. J. (2013). Exploration, novelty, surprise, and free energy minimization. Frontiers in Psychology, 4:710. doi:10.3389/fpsyg.2013.00710

Seth, A. K., Suzuki, K., and Critchley, H. D.(2012), An interoceptive predictive coding model of conscious presence. Frontiers in Psychology, 2:395. doi: 10.3389/fpsyg.2011.0039.

Seth A. K. (2014). A predictive processing theory of sensorimotor contingencies: Explaining the puzzle of perceptual presence and its absence in synesthesia. Cognitive Neuroscience, 5:2, 97-118.

Clark, A. (2016). Surfing uncertainty: Prediction, action, and the embodied mind. Oxford: Oxford University Press.

Miyahara, K., Niikawa, T., Hamada, H. T., and Nishida, S. (2020). Developing a short-term phenomenological training program: A report of methodological lessons. New Ideas in Psychology, 58, 100780. doi: 10.1016/j.newideapsych.2020.100780

Kordeš, U. (2016). Going beyond theory: Constructivism and empirical phenomenology. Constructivist Foundations, 14:2, 149152.

Petitmengin, C., Navarro, V., and Le Van Queyen, M. (2007). Anticipating seizure: Pre-reflective experience at the center of neuro-phenomenology. Consciousness and Cognition, 16:3, 746-764.

Schwartzman, D. J., Oblak, A., Rothen, N., Bor, D., and Seth, A. K. (2020). Extensive Phenomenological Overlap between Induced and Naturally-Occurring Synaesthetic Experiences. BioRxiv, 228692. doi: $10.1101 / 2020.08 .03 .228692$

Nelson J. (2017) Using conceptual depth criteria: Addressing the challenge of reaching saturation in qualitative research. Qualitative Research 17:5, 554-570.

Saunders, B., Sim, J. Kingstone, T., Baker, S., Waterfield, J., Bartlam, B., Burroughs, H., and Jinks, C. (2018). Saturation in qualitative research: exploring its conceptualization and operationalization. Quality \& Quantity, 52:4, 1893-1907. doi: 10.1007/s11135-017-0574-8.

Colombetti, G. (2011). Varieties of Pre-Reflective Self-Awareness: Foreground and Background Bodily Feelings in Emotion Experience. Inquiry: An Interdisciplinary Journal of Philosophy, 54:3, 293-313.

Kordeš, U. (2019). Horizons of Analysis. Constructivist Foundations, 14:2, 149-152.

Mogensen, J., and Overgaard, M. (2018). Reorganization of the connectivity between elementary functions as a common mechanism of phenomenal consciousness and working memory: from functions to strategies. Philosophical Transactions of the Royal Society of London. Series B, Biological Sciences , 373:1755, 20170346. doi: 10.1098/rstb.2017.0346.

Petitmengin, C. (2017). Enaction as a lived experience: Towards a radical neurophenomenology. Constructivist Foundations 12:2, 139-147. 
Orne, M. (1962). On the social psychology of the psychological experiment: With particular reference to demand characteristics and their implications. American Psychologist, 17:1, 776-783.

Hurlburt R. T., Alderson-Day, B., Kühn, S., and Fernyhough, C. (2016). Exploring the Ecological Validity of Thinking on Demand: Neural Correlates of Elicited vs. Spontaneously Occurring Inner Speech. PLoS ONE, 11:2, e0147932. doi: 10.1371/journal.pone.0147932

Fernyhough, C., Alderson-Day, B., Hurlburt, R. T. and Kühn, S. (2018). Investigating Multiple Streams of Consciousness: Using Descriptive Experience Sampling to Explore Internally and Externally Directed Streams of Thought. Frontiers in Human Neuroscience 12:494. doi: 10.3389/fnhum.2018.00494.

Lutz, A., Lachaux, J., Martinerie, J., and Varela, F. J. (2002). Guiding the study of brain dynamics by using first-person data: Synchrony patterns correlate with ongoing conscious states during a simple visual task. PNAS, 99:3, 1586-1591.

Gallagher, S., and Brøsted Sørensen, J. (2006). Experimenting with phenomenology. Consciousness and Cognition, 15:1, 119-134.

Charmaz, K., and Belgrave, L. L. (2007). Grounded theory. The Blackwell encyclopedia of sociology.

Mills, J., Bonner, A., and Francis, K. (2006a). Adopting a constructivist approach to grounded theory: Implications for design. International Journal of Nursing Practice, 12:1, 8-13.

Mills, J., Bonner, A., and Francis, K. (2006b). The development of constructivist grounded theory. International Journal of Qualitative Methods, 5:1, 25-35.

Petitmengin, C. (2006). Describing one's subjective experience in the second person: An interview method for the science of consciousness. Phenomenology and the Cognitive Sciences, 5:1, 229-269.

Hurlburt, R. T. (2011). Investigating Pristine Inner Experience: Moments of Truth. Cambridge:The MIT Press.

Kordeš, U., and Klauser, F. (2016). Second-person in-depth phenomenological inquiry as an approach for studying enaction of beliefs. Interdisciplinary Description of Complex Systems, 14:4, 369-377.

Petitmengin, C., Remillieux, A., and Valenzuela-Moguillansky, C. (2019). Discovering the structures of lived experience. Phenomenology and the Cognitive Sciences, 18:4, 691-730.

Charmaz, K. (2004). Constructing Grounded Theory: A Practical Guide Through Qualitative Analysis . London: Sage Publications.

Flick, U. (2009). An Introduction to Qualitative Analysis . London: Sage Publications.

Kalinowski, P., Lai, J., Fidler, F., and Cumming, G. (2010). Qualitative Research: An Essential Part of Statistical Cognition Research. Statistics Education Research Journal , 9:2, 22-34. 
Hurlburt, R. T., and Heavey, C. L. (2006). Exploring inner experience: The descriptive experience sampling method. Amsterdam: John Benjamins.

Kordeš, U., Oblak, A., Smrdu, M., and Demšar, E. (2019). Ethnography of meditation: An account of pursuing meditative practice as a tool for researching consciousness. Journal of Consciousness Studies, 26: 7-8), 184-237.

Valenzuela-Moguillansky, C., and Vásquez-Rosati, A. (2019). An analysis procedure for the micro-phenomenological interview. Constructivist Foundations, 14:2, 123-145.

Morisson, H., McBriar, S., Powell, H., Proudfoot, J., Stanley, S., Fitzgerald, D., Callard, F. (2019). What is a Psychological Task? The Operational Pliability of "Task" in Psychological Laboratory Experimentation. Engaging Science, Technology, and Society. 5, 61-85.

Jaspers, K. (1997). General Psychopathology, Volume 1. Baltimore: Johns Hopkins University Press.

Seghier, M. L., and Price, C. J. (2018). Interpreting and utilising intersubject variability in brain function. Trends in Cognitive Sciences. 22:6, 517-530.

D'Angiuli, A., and LeBeau, L. S., (2002). On boredom and experimentation in humans. Ethics \& Behavior. 12:2, 167-76.

Ikeda, M., Iwanaga M., Seiwa, H. (1996). Test Anxiety and Working Memory System. Perceptual and Motor Skills. 82:3, 1223-1231

Varela, F. J., and Shear, J. (1999). First-person methodologies: What, why, how? Journal of Consciousness Studies, 6:1, 1-14.

Lutz, A., Lachaux, J., Martinerie, J., Varela, F. J. (2002). Guiding the study of brain dynamics by using first-person data: Synchrony patterns correlate with ongoing conscious states during a simple visual task. PNAS. 99:3, 1586-1591.

Fernyhough, C., Alderson-Day, B., Hurlburt, R. T., Kühn, S (2018). Investigating Multiple Streams of Consciousness: Using Descriptive Experience Sampling to Explore Internally and Externally Directed Streams of Thought. Frontiers in Human Neuroscience 12. doi: 10.3389/fnhum.2018.00494.

Hurlburt R. T., Alderson-Day, B., Kühn, S., Fernyhough, C. (2016). Exploring the Ecological Validity of Thinking on Demand: Neural Correlates of Elicited vs. Spontaneously Occurring Inner Speech. PLoS ONE, 11:2, e0147932. doi: 10.1371/journal.pone.0147932

Buchsbaum, B. R. (2013). The role of consciousness in the phonological loop: hidden in plain sight. Front. Psychology, 4:496. doi: 10.3389/fpsyg.2013.00496.

Oblak, A. (2020). Visual representation in the wild: Empirical phenomenological investigation of visual-spatial working memory in a naturalistic setting. Constructivist Foundations. 15:3, 238-250. 
Bo, J., and Seidler, R. D. (2009). Visuospatial Working Memory Capacity Predicts the Organization of Acquired Explicit Motor Sequences. Journal of Neurophysiology. 101:6, 3116-3125.

Peirce, J.W. (2007). PsychoPy - Psychophysics software in Python. Journal of Neuroscience Methods. 162:1-2, 8-13. 